\title{
DIFFERENTIAL DELAY EQUATIONS THAT HAVE PERIODIC SOLUTIONS OF LONG PERIOD
}

\author{
STEVEN A. CHAPIN
}

\begin{abstract}
If $f: \mathbf{R} \rightarrow \mathbf{R}$ is a continuous odd function satisfying $x f(x)>0$, $x \neq 0$, and $f(x)=o\left(x^{-2}\right)$ as $x \rightarrow \infty$, then so-called periodic solutions of long period seem to play a prominent role in the dynamics of
\end{abstract}

$$
x^{\prime}(t)=-\alpha f(x(t-1)), \quad \alpha>0 .
$$

In this paper we prove the existence of long-period periodic solutions of $(*)$ for a class of nonodd functions that decay "rapidly" to 0 at infinity and satisfy $x f(x) \geq 0$. These solutions have quite different qualitative features than in the odd case.

Introduction. Consider the parametrized differential delay equation

$$
x^{\prime}(t)=-\alpha f(x(t-1)), \quad \alpha>0,
$$

where $f: \mathbf{R} \rightarrow \mathbf{R}$ is a continuous nonlinear function. A nonconstant periodic solution $x(t)$ of $(0.1)$ will be called a slowly oscillating periodic solution if there exist numbers $q>1$ and $q^{\prime}>q+1$ such that $x(t)>0$ on $(0, q), x(t)<0$ on $\left(q, q^{\prime}\right)$, and $x\left(t+q^{\prime}\right)=x(t)$ for all $t$ (thus, $q^{\prime}$ is the minimal period of $x(t)$ ). The word "slowly" refers to the fact that the zeros of $x(t)$ are separated by a distance greater than the delay in (0.1), which is 1 . If $f$ satisfies $x f(x)>0, x \neq 0$, then slowly oscillating periodic solutions are of fundamental importance in understanding the dynamics of $(0.1)[15]$. (The assumption $x f(x)>0, x \neq 0$, is fairly standard in relation to $(0.1)$ and typically causes solutions to oscillate around $x=0$.)

Now, suppose that there exists an $\alpha_{0}>0$ such that for every $\alpha \geq \alpha_{0},(0.1)$ has a slowly oscillating periodic solution $x_{\alpha}(t)$ such that the minimal period of $x_{\alpha}(t)$ tends to infinity as $\alpha \rightarrow \infty$. Then we will say that $(0.1)$ has periodic solutions of long period. The class of functions for which long-period periodic solutions of $(0.1)$ exist is certainly large (see, for example, $[\mathbf{1 0}]$ ) and is known to contain some classes of functions $f$ for which $x f(x)>0, x \neq 0$, and $\lim _{x \rightarrow \infty} f(x)=0$. In examples of this type for which the existence of long period periodic solutions has been proved numerical experiments suggest that they have strong stability properties, though, to the best of our knowledge, nothing has been proved in this area. We should also mention that these $f$ are not monotone and that there is ample evidence by now that if $f$ is not monotone, then the dynamics of $(0.1)$ can be quite complex (see, for example, [12-14]).

The first theorems concerning the existence of long-period periodic solutions, for functions decaying to 0 at $\infty$, were proved under the assumption that $f$ was an

Received by the editors November 3, 1986.

1980 Mathematics Subject Classification (1985 Revision). Primary 34K25. 
odd function $(f(-x)=-f(x)$ for all $x)$. For example, R. D. Nussbaum studied equation (0.1) with $f$ given by

$$
f(x)=x\left(1+|x|^{r+1}\right)^{-1}, \quad r>0 .
$$

He was motivated by the observation that, at least in some respects, the dynamics of $(0.1)$ with this $f$ seern similar to the dynamics of an equation proposed by Mackey and Glass in a study of the production of blood cells [6]. Nussbaum proved that, for $f$ given by (0.2), if $r>2$ (somewhat more general $f$ is allowed, but $f$ must be odd), then equation (0.1) has periodic solutions of long period [7]; moreover, he showed that $r=2$ is a cutoff in the sense that if $r<2$, then $(0.1)$ does not have long-period periodic solutions. (This author showed that long-period periodic solutions do exist for the case $r=2$ [2].)

Although the assumption that $f$ decays like $x^{-r}$ at $\infty$ plays a role in the proof in [7], numerical studies suggest that long-period periodic solutions exist for odd functions which decay more rapidly than $x^{-r}$ for all $r$, e.g., functions that decay exponentially at $\infty$. In fact, it has been conjectured [9] that if $f$ satisfies

(i) $f$ is odd $(f(-x)=-f(x)$ for all $x)$;

(ii) $x f(x)>0, x \neq 0$;

(iii) $f \mid\left[0, x_{*}\right]$ is increasing (for some $x_{*}>0$ ), and $f \mid\left[x_{*}, \infty\right)$ is decreasing;

(iv) $\lim _{x \rightarrow \infty} x^{2} f(x)=0$,

then (0.1) has long-period periodic solutions. This conjecture remains unproved, though this author has been able to prove the existence of long-period periodic solutions for a class of functions satisfying (i)-(iii) and which decay exponentially, e.g., $f(x)=x \exp \left(-b x^{2}\right), b>0[1]$.

We strongly believe that this conjecture is true; however, for examples satisfying (i)-(iv) for which the existence of long-period periodic solutions has been proved the asymptotic behavior of the minimal period (as a function of $\alpha$ ) depends on the asymptotic behavior of $f$. Recall that $q_{\alpha}^{\prime}=q^{\prime}(\alpha)$ is the minimal perriod of $x_{\alpha}(t)$. Then one has for $f$ given by $(0.2)$

$$
\begin{gathered}
c_{1} \alpha^{r-2} \leq q_{\alpha}^{\prime} \leq c_{2} \alpha^{r-2}, \quad r>2, \\
c_{1}(\log \alpha)^{3} \leq q_{\alpha}^{\prime} \leq c_{2}(\log \alpha)^{3}, \quad r=2,
\end{gathered}
$$

where $c_{1}$ and $c_{2}$ are positive constants depending on $r$ but not on $\alpha$. This is proved in [4] for $r>2$ and [2] for $r=2$. For the example, $f(x)=x \exp \left(-b x^{2}\right), b>0$, one can show there exists a positive constant $\gamma$ (independent of $\alpha$ ) such that

$$
q_{\alpha}^{\prime} \geq \exp \left(\alpha^{\gamma}\right) \quad[1]
$$

In this paper we are interested in long-period periodic solutions to $(0.1)$ where $f$ is not odd. We prove the existence of long-period periodic solutions with very different qualitative features than in the odd case. In the examples mentioned above the long-period periodic solutions exhibit the symmetry $x_{\alpha}\left(t+q_{\alpha}\right)=-x_{\alpha}(t)$ for all $t$ (so $q_{\alpha}^{\prime}=2 q_{\alpha}$ ). This symmetry does not exist in the nonodd case, and in fact we will see the $\lim _{\alpha \rightarrow \infty} q_{\alpha}^{\prime}=\infty$, but $q_{\alpha}^{\prime}-q_{\alpha}<2$. In [1] this author proved the existence of long-period periodic solutions, with the qualitative features described above for functions that satisfy $x f(x)>0, x \neq 0 ; f$ is increasing on $\left[-x_{1}, x_{2}\right]$, $x_{1}, x_{2}>0 ; f$ is decreasing on $\left(-\infty,-x_{1}\right]$ and on $\left[x_{2}, \infty\right) ; f(x)=a x^{-r}+o\left(x^{-r}\right)$ as 
$x \rightarrow \infty, a>0, r>1 ;$ and

$$
\int_{0}^{\infty} f(x) d x<-\int_{-\infty}^{0} f(x) d x \leq \infty
$$

The proof is long and fairly technical, and so rather than give this proof we choose to consider hypotheses on $f$ which make the proof somewhat simpler (although still fairly long). The basic ideas, though, are the same. Among the allowable functions are

$$
f(x)=x^{2 n+1} e^{-\lambda x}, \quad \lambda>0, n=0,1,2, \ldots .
$$

If $f(x)=x e^{-\lambda x}, \lambda>0$, then our equation has formally the same relationship to an equation in population dynamics as Nussbaum's equation has to the Mackey and Glass equation [5].

Finally, we should mention that if $f^{\prime}(0)>0, x f(x)>0, x \neq 0$, and $f$ is bounded above (or below), then (0.1) has a slowly oscillating periodic solution for $\alpha>\pi /\left(2 f^{\prime}(0)\right)[8]$. These results however (see also [7]) give no information about the minimal period of these solutions for large $\alpha$ if $\lim _{x \rightarrow \infty} f(x)=0$. Moreover, we allow the possibility that $f^{\prime}(0)=0$, e.g., $f(x)=x^{3} e^{-\lambda x}, \lambda>0$, or that $f^{\prime}(0)$ does not exist.

1. Preliminaries and the main result. Throughout this paper we will assume that $f$ is a function that satisfies the following:

(H) $f: \mathbf{R} \rightarrow \mathbf{R}$ is continuous, $x f(x)>0$ for $x \neq 0$, and there exist positive constants $x_{*}, a_{1}, a_{2}, \lambda$, and a real constant $r$ such that

(i) $f \mid\left(-\infty, x_{*}\right]$ is nondecreasing and $f \mid\left[x_{*}, \infty\right)$ is nonincreasing, and

(ii) $a_{1} x^{r} e^{-\lambda x} \leq f(x) \leq a_{2} x^{r} e^{-\lambda x}, x \geq x_{*}$.

In addition, there exist a positive constant $x_{0}$ and a constant $d>2 f\left(x_{*}\right) \lambda a_{2} / a_{1}$ $\left(a_{1}, a_{2}, x_{*}\right.$, and $\lambda$ as above) such that

$$
f(x) \leq d x, \quad x \leq-x_{0} .
$$

Henceforth, the symbols $x_{*}, a_{1}, a_{2}, \lambda, r, x_{0}$, and $d$ will always be taken as in condition $(\mathrm{H})$.

REMARK 1.1. The functions

$$
f(x)=x^{2 n+1} e^{-\lambda x}, \quad n=0,1,2, \ldots
$$

satisfy condition $(\mathrm{H})$.

The precise theorem we will prove is as follows.

THEOREM. Suppose that $f$ satisfies $(\mathrm{H})$. Then there exists an $\alpha_{0}>0$ such that for $\alpha \geq \alpha_{0}$ equation (0.1) has a nonconstant periodic solution $x_{\alpha}(t)$. Moreover, for $\alpha \geq \alpha_{0}, x_{\alpha}(t)$ has the following properties:

(i) there exist numbers $q_{\alpha}>1$ and $q_{\alpha}^{\prime}>q_{\alpha}+1$ such that $x_{\alpha}(t)>0$ on $\left(0, q_{\alpha}\right)$, $x_{\alpha}(t)<0$ on $\left(q_{\alpha}, q_{\alpha}^{\prime}\right)$, and $x_{\alpha}(t)=x_{\alpha}\left(q_{\alpha}^{\prime}+t\right)$ for all $t\left(q_{\alpha}^{\prime}\right.$ is the minimal period of $\left.x_{\alpha}(t)\right)$;

(ii) there exists a positive constant $\beta$, independent of $\alpha$, such that

$$
q_{\alpha}^{\prime} \geq \exp (\beta \alpha) ; \quad \text { and }
$$

(iii) $q_{\alpha}^{\prime}-q_{\alpha}<2$.

The proof of existence of periodic solutions is accomplished by estimating (in terms of $\alpha$ ) the size of certain solutions of (0.1) over certain intervals. A fairly 
standard application of Schauder's fixed point theorem then implies the existence of periodic solutions satisfying properties (i) and (iii) of the theorem. This is the content of $\S 2$. In $\S 3$ we prove the asymptotic estimate given by (ii) of the theorem.

In the sequel the phrases "for a sufficiently large" and "for $\alpha$ large enough" mean "there exists an $\alpha_{0}$ such that for $\alpha \geq \alpha_{0}$ ".

2. Existence of periodic solutions. Consider the differential delay equation

$$
x^{\prime}(t)=-\alpha f(x(t-1)), \quad \alpha>0 .
$$

Suppose $f: \mathbf{R} \rightarrow \mathbf{R}$ and $\varphi:[0,1] \rightarrow \mathbf{R}$ are continuous. Then it is well known that (2.1) has a unique solution $x(t)=x(t ; \varphi, \alpha), t \geq 0$, such that $x(t)$ is continuous, $x \mid[0,1]=\varphi$, and $x(t)$ satisfies $(2.1)$ for $t>1$. This solution can be explicitly obtained by integration:

$$
\begin{aligned}
x(t) & =x(1)-\alpha \int_{1}^{t} f(x(s-1)) d s \\
& =\varphi(1)-\alpha \int_{0}^{t-1} f(\varphi(s)) d s, \quad 1 \leq t \leq 2 .
\end{aligned}
$$

Now $x(t)$ is known on $[1,2]$, so we may continue:

$$
\begin{aligned}
x(t) & =x(2)-\alpha \int_{2}^{t} f(x(s-1)) d s \\
& =x(2)-\alpha \int_{1}^{t-1} f(x(s)) d s, \quad 2 \leq t \leq 3 .
\end{aligned}
$$

Continuing in this manner one obtains $x(t)$ on $[0, \infty)$.

Let $C[0,1]$ denote the real Banach space of continuous real-valued functions on $[0,1]$ equipped with the usual supremum norm, and define

$$
K_{\alpha, k}=\left\{\varphi \in C[0,1] \mid \varphi(0) \leq-\alpha f\left(-\alpha f\left(x_{*}\right)\right),\right.
$$

$$
\varphi \text { is nonincreasing, } \varphi(1)=k \log \alpha\} \text {. }
$$

Note that for a fixed $k, K_{\alpha, k}$ is nonempty if $\alpha$ is sufficiently large, and is a closed, bounded, convex subset of $C[0,1]$. Given $\alpha, \varphi \in C[0,1], x(t)=x(t ; \varphi, \alpha)$ will always denote the solution to (2.1) described at the beginning of this section.

The main purpose of the first two lemmas is to estimate $\alpha$ in terms of quantities that, it turns out, will be easier to work with. Here we follow fairly closely the work of Nussbaum in [7].

LEMMA 2.1. Fix $k>2 / \lambda$. If $\alpha$ is sufficiently large and $\varphi \in K_{\alpha, k}$, then one has

$$
z_{1} \underset{\text { def. }}{=} \inf \{t>0 \mid x(t)=0\} \geq 3, \quad \text { and } \quad x\left(z_{1}-1\right) \geq x_{*} .
$$

PROOF. Since $\varphi$ is nonincreasing and $f$ is nonincreasing on $\left[x_{*}, \infty\right)$ one has

$$
\begin{aligned}
x^{\prime}(t) & \geq-\alpha f(\varphi(1)) \geq-\alpha a_{2}(k \log \alpha)^{r} e^{-k \lambda \log \alpha} \\
& =-a_{2}(k \log \alpha)^{r} \alpha^{1-k \lambda}, \quad 1 \leq t \leq 2
\end{aligned}
$$

It follows that

$$
\begin{aligned}
x(2) & \geq k \log \alpha-a_{2}(k \log \alpha)^{r} \alpha^{1-k \lambda} \\
& =k \log \alpha\left(1-a_{2}(k \log \alpha)^{r-1} \alpha^{1-k \lambda}\right) \geq \frac{k}{2} \log \alpha
\end{aligned}
$$

for $k \lambda>1$ and $\alpha$ sufficiently large. 
Similarly $(x(t)$ is decreasing on $[1,2])$,

$$
\begin{aligned}
x^{\prime}(t) & \geq-\alpha f(x(2)) \geq-\alpha a_{2}\left(\frac{k}{2} \log \alpha\right)^{r} e^{-(k / 2) \lambda \log \alpha} \\
& =-a_{2}\left(\frac{k}{2} \log \alpha\right)^{r} \alpha^{1-(k / 2) \lambda}, \quad 2 \leq t \leq 3 .
\end{aligned}
$$

Thus,

$$
x(3) \geq \frac{k}{2} \log \alpha-a_{2}\left(\frac{k}{2} \log \alpha\right)^{r} \alpha^{1-(k / 2) \lambda} \geq 0
$$

for $(k / 2) \lambda>1$ and $\alpha$ sufficiently large.

This proves that $z_{1} \geq 3$.

To prove the next part suppose, for the sake of obtaining a contradiction, that $x\left(z_{1}-1\right)<x_{*}$, and define

$$
t_{1}=\inf \left\{t>0 \mid x(t)=x_{*}\right\} .
$$

$x(t)$ is concave down and decreasing on $\left[1, t_{1}+1\right]$. $(x(t)$ is concave down on an interval will simply mean $x^{\prime}(t)$ is nonincreasing on that interval; similarly for concave up.) This implies

$$
\left|x^{\prime}\left(t_{1}\right)\right| \leq x\left(t_{1}\right)-x\left(t_{1}-1\right) \leq x_{*} .
$$

It follows that

$$
x\left(t_{1}-1+s\right) \leq x_{*}+(1-s) x_{*}, \quad 0 \leq s \leq 1 .
$$

Therefore, integrating the differential equation and substituting gives

$$
\begin{aligned}
x\left(t_{1}+1\right) & =x_{*}-\alpha \int_{0}^{1} f\left(x\left(t_{1}-1+s\right)\right) d s \\
& \leq x_{*}-\alpha \int_{0}^{1} f\left(x_{*}+(1-s) x_{*}\right) d s \\
& =x_{*}-\left(\frac{\alpha}{x_{*}}\right) \int_{x_{*}}^{2 x_{*}} f(u) d u .
\end{aligned}
$$

Now, we are assuming $x\left(t_{1}+1\right)>0$, so we must have

$$
\alpha<x_{*}^{2}\left[\int_{x_{*}}^{2 x *} f(u) d u\right]^{-1} .
$$

Since $f(x)>0$ on $\left[x_{*}, 2 x_{*}\right]$, this is impossible for $\alpha$ sufficiently large, so we conclude that $x\left(z_{1}-1\right) \geq x_{*}$. (Note that here we have proved that $x\left(t_{1}+1\right)<0$. It is easy to see that $t_{1}<\infty$, hence $z_{1}<\infty$.)

Choose a fixed $k^{\prime}>2 / \lambda$, and define $K_{\alpha}=K_{\alpha, k^{\prime}}$. Also, given $\alpha$ and $\varphi \in K_{\alpha}$ define

$$
\begin{gathered}
z_{1}=\inf \{t>0 \mid x(t)=0\}, \quad m=x\left(z_{1}-1\right), \\
\delta=\inf \left\{t>0 \mid x\left(z_{1}-t\right)=x_{*}\right\}
\end{gathered}
$$

If $\alpha$ is sufficiently large, it follows from Lemma 2.1 that all of these numbers exist and are finite. In the sequel we will always implicitly assume that $\alpha$ is large enough so that all of the previous results hold. 
LEMMA 2.2. Let $c_{1}>\lambda a_{2} / a_{1}$ be a constant $\left(a_{1}, a_{2}\right.$ as in $\left.(\mathrm{H})\right)$. If $\alpha$ is suffciently large and $\varphi \in K_{\alpha}$, then

$$
\begin{gathered}
m / f(m) \leq \alpha \leq c_{1} m^{2} / f(m), \quad \text { and } \\
x_{*} / \alpha f(m) \leq \delta \leq x_{*} / m .
\end{gathered}
$$

PROOF. Since $x(t)$ is decreasing on $\left[z_{1}-2, z_{1}-1\right]$,

$$
m=\alpha \int_{z_{1}-2}^{z_{1}-1} f(x(s)) d s \leq \alpha f(m), \quad \text { or } \quad \frac{m}{f(m)} \leq \alpha .
$$

To obtain the required upper bound for $\alpha$, we will first prove a cruder estimate which implies that $m \rightarrow \infty$ as $\alpha \rightarrow \infty$. Since $x(t)$ is concave down on $\left[z_{1}-2, z_{1}\right]$, we have $x\left(z_{1}-2\right) \leq 2 m$. Using this estimate, the concavity of $x(t)$, and the properties of $f$, one obtains

$$
\begin{aligned}
m & \geq-x^{\prime}\left(z_{1}-1\right)=\alpha f\left(x\left(z_{1}-2\right)\right) \geq \alpha f(2 m) \\
& \geq \alpha a_{1}(2 m)^{r} e^{-\lambda(2 m)}, \quad \text { or } \\
\alpha & \leq a_{1}^{-1} 2^{-r} m^{1-r} e^{2 \lambda m} .
\end{aligned}
$$

Lemma 2.1 implies that $m \geq x_{*}$, so we must have $m \rightarrow \infty$ as $\alpha \rightarrow \infty$.

Again using the concavity of $x(t)$ and the estimate on $x\left(z_{1}-2\right)$ we find that

$$
\begin{aligned}
m & =\alpha \int_{z_{1}-2}^{z_{1}-1} f(x(s)) d s \\
& \geq \frac{\alpha}{m} \int_{m}^{2 m} f(u) d u \quad\left(x^{\prime}(s) \geq-m \text { on }\left[z_{1}-2, z_{1}-1\right]\right) \\
& \geq\left(\frac{\alpha}{m}\right) a_{1} \int_{m}^{2 m} u^{r} e^{-\lambda u} d u \\
& =\left(\frac{\alpha}{m}\right) a_{1}\left[\frac{m^{r} e^{-\lambda m}}{\lambda}-\frac{2^{r} m^{r} e^{-2 \lambda m}}{\lambda}+\frac{r}{\lambda} \int_{m}^{2 m} u^{r-1} e^{-\lambda u} d u\right] \\
& =\left(a_{1} / \lambda\right) \alpha m^{r-1} e^{-\lambda m}[1+g(m)] \\
& \geq\left(a_{1} / \lambda a_{2}\right) \frac{\alpha f(m)}{m}[1+g(m)],
\end{aligned}
$$

where

$$
g(m)=\frac{e^{\lambda m}}{m^{r}}\left[r \int_{m}^{2 m} u^{r-1} e^{-\lambda u} d u-2^{r} m^{r} e^{-2 \lambda m}\right] .
$$

It is not difficult to see that $g(m) \rightarrow 0$ as $m \rightarrow \infty$, hence $g(m) \rightarrow 0$ as $\alpha \rightarrow$ $\infty$. Choose $c_{1}>\lambda a_{2} / a_{1}$. It follows that, if $\alpha$ is sufficiently large, one has $m \geq$ $\alpha f(m) / c_{1} m$. This gives the other part of $(2.2)$.

To prove (2.3) recall that Lemma 2.1 implies $\delta \leq 1$. Since $x(t)$ is concave down on $\left[z_{1}-1, z_{1}\right]$, we have

$$
-x^{\prime}(t) \leq-x^{\prime}\left(z_{1}\right)=\alpha f(m), \quad z_{1}-\delta \leq t \leq z_{1} .
$$

The mean-value theorem implies that

$$
-x^{\prime}(\xi)=\left(x\left(z_{1}-\delta\right)-x\left(z_{1}\right)\right) / \delta=x_{*} / \delta \quad \text { for some } \xi \in\left(z_{1}-\delta, z_{1}\right) .
$$


This implies the inequality $x_{*} / \delta \leq \alpha f(m)$, or $x_{*} / \alpha f(m) \leq \delta$.

For the other part of (2.3) we use again the fact that $x(t)$ is concave down on $\left[z_{1}-1, z_{1}\right]$. This implies that

$$
\frac{m-x_{*}}{1-\delta} \leq m, \quad \text { or } \quad \delta \leq \frac{x_{*}}{m}
$$

Before proceeding further, given $\alpha$ and $\varphi \in K_{\alpha}$, let us define functions $\psi_{0}, \psi_{1}$, and $\psi_{2}$ as follows.

$$
\begin{aligned}
& \psi_{0}(t)=x\left(z_{1}-1+t\right), \quad 0 \leq t \leq 1 \\
& \psi_{1}(t)=\alpha \int_{0}^{t} f\left(\psi_{0}(s)\right) d s, \quad 0 \leq t \leq 1 \\
& \psi_{2}(t)=-\alpha \int_{0}^{t} f\left(-\psi_{1}(s)\right) d s, \quad 0 \leq t \leq 1
\end{aligned}
$$

Note that

$$
\psi_{1}(1)=\left|x\left(z_{1}+1\right)\right|, \quad \text { and } \quad x\left(z_{1}+2\right)=-\psi_{1}(1)+\psi_{2}(1) .
$$

Our next immediate goal is to estimate $x\left(z_{1}+2\right)$.

LEMMA 2.3. Let $c_{2}<1$ be a positive constant. If $\alpha$ is sufficiently large, $\varphi \in K_{\alpha}$, then

$$
\frac{c_{2}}{f(m)} \int_{0}^{\infty} f(\xi) d \xi \leq \psi_{1}(1) \leq \alpha f\left(x_{*}\right) .
$$

ProOF. $\psi_{1}(1) \leq \alpha f\left(x_{*}\right)$ is trivial, since

$$
\left|x^{\prime}(t)\right| \leq \alpha f\left(x_{*}\right), \quad z_{1} \leq t \leq z_{1}+1 .
$$

For the other part, we start with the observation that $\psi_{0}(s)$ is concave down on $[0,1]$, so we have

$$
\psi_{0}(s) \geq-\frac{x_{*}}{\delta}(s-1), \quad 1-\delta \leq s \leq 1
$$

Since $f$ is nondecreasing on $\left[0, x_{*}\right]$, we obtain

$$
\begin{aligned}
\alpha \int_{1-\delta}^{1} f\left(\psi_{0}(s)\right) d s & \geq \alpha \int_{1-\delta}^{1} f\left(\frac{x_{*}}{\delta}(1-s)\right) d s \\
& =\left(\frac{\alpha \delta}{x_{*}}\right) \int_{0}^{x_{*}} f(u) d u \\
& \geq \frac{1}{f(m)} \int_{0}^{x_{*}} f(u) d u
\end{aligned}
$$

(Here we have used (2.3) and the obvious substitution.) Again using the concavity of $\psi_{0}(s)$, we observe that

$$
\begin{aligned}
& \psi_{0}(s) \leq x_{*}+(1-\delta-s)\left|\psi_{0}^{\prime}(0)\right| \\
& \leq x_{*}+(1-\delta-s) \alpha f(m) \\
&=\theta_{0}(s), \quad 0 \leq s \leq 1-\delta \\
& \text { def. }
\end{aligned}
$$


Using the fact that $f$ is nonincreasing on $\left[x_{*}, \infty\right)$ we obtain

$$
\begin{aligned}
\psi_{1}(1-\delta) & \geq \alpha \int_{0}^{1-\delta} f\left(\theta_{0}(s)\right) d s \\
& =\frac{1}{f(m)} \int_{x_{*}}^{x_{*}+(1-\delta) \alpha f(m)} f(u) d u
\end{aligned}
$$

The estimates in Lemma 2.2 imply that there exists a positive constant $k$ (independent of $\alpha$ and $\left.\varphi \in K_{\alpha}\right)$ such that $x_{*}+(1-\delta) \alpha f(m) \geq k m$. It follows that

$$
\begin{aligned}
\psi_{1}(1-\delta) & \geq \frac{1}{f(m)} \int_{x_{*}}^{k m} f(u) d u \\
& =\frac{1}{(f(m)}\left[\int_{x_{*}}^{\infty} f(u) d u-\int_{k m}^{\infty} f(u) d u\right]
\end{aligned}
$$

The proof of the lemma is now easily completed using $(2.5),(2.6)$, and the properties of $f$.

LEMMA 2.4. Let $d_{1}<d / 2$ be a positive constant ( $d$ as in $\left.(\mathrm{H})\right)$. If $\alpha$ is sufficiently large, $\varphi \in K_{\alpha}$, then

$$
\psi_{2}(1) \geq d_{1} m^{2} / f(m) .
$$

Proof. Note that $\psi_{1}(t)=-x\left(z_{1}+t\right), 0 \leq t \leq 1$, so $\psi_{1}(t)$ is concave up on $[0,1-\delta]$. It follows that

$$
\psi_{1}(t) \geq t \alpha f(m), \quad 0 \leq t \leq 1-\delta .
$$

Since $f$ is nondecreasing on $(-\infty, 0]$, we obtain

$$
\begin{aligned}
-\alpha \int_{0}^{1-\delta} f\left(-\psi_{1}(t)\right) d t & \geq-\alpha \int_{0}^{1-\delta} f(-t \alpha f(m)) d t \\
& =-\frac{1}{f(m)} \int_{0}^{\alpha f(m)(1-\delta)} f(-u) d u \\
& \geq-\frac{1}{f(m)} \int_{0}^{m-m \delta} f(-u) d u
\end{aligned}
$$

(Here we have made the substitution $u=t \alpha f(m)$ and then used (2.2).)

The assumption that $f$ satisfies $(\mathrm{H})$ implies that

$$
\begin{aligned}
-\frac{1}{f(m)} \int_{0}^{m-m \delta} f(-u) d u & \geq-\frac{1}{f(m)}\left[\int_{0}^{x_{0}} f(-u) d u-d \int_{x_{0}}^{m-m \delta} u d u\right] \\
& =-\frac{1}{f(m)}\left[\int_{0}^{x_{0}} f(-u) d u-\frac{d(m-m \delta)^{2}}{2}+\frac{d x_{0}^{2}}{2}\right] \\
& =\left(\frac{d}{2}\right) \frac{m^{2}}{f(m)}\left[\frac{-2}{m^{2} d} \int_{0}^{x_{0}} f(-u) d u+(1-\delta)^{2}-\frac{x_{0}^{2}}{m^{2}}\right] .
\end{aligned}
$$

The lemma now follows from Lemma 2.2 (the estimate on $\delta$ ), the fact that $\int_{0}^{x_{0}} f(-u) d u$ is bounded by a fixed constant, independent of $\alpha, \varphi$, and the observation that $\psi_{2}(1) \geq-\alpha \int_{0}^{1-\delta} f\left(-\psi_{1}(t)\right) d t$. We leave the exact details to the reader. 
LEMMA 2.5. If $\alpha$ is sufficiently large, $\varphi \in K_{\alpha}$, then there is a positive constant $d_{2}$ (independent of $\alpha$ and $\varphi \in K_{\alpha}$ ) such that $x\left(z_{1}+2\right) \geq d_{2} m^{2} / f(m)$.

PrOOF. Choose positive constants $c_{1}^{\prime}$ and $d_{1}^{\prime}$ satisfying

$$
f\left(x_{*}\right) \lambda a_{2} / a_{1}<c_{1}^{\prime} f\left(x_{*}\right)<d_{1}^{\prime}<d / 2 .
$$

$\left(a_{1}, a_{2}, d, \lambda, x_{*}\right.$ as in (H). Recall $d>2 f\left(x_{*}\right) \lambda a_{2} / a_{1}$, so this is possible.)

It follows, using Lemmas 2.2-2.4, with $c_{1}=c_{1}^{\prime}$ and $d_{1}=d_{1}^{\prime}$, that

$$
\begin{aligned}
x\left(z_{1}+2\right) & =-\psi_{1}(1)+\psi_{2}(1) \\
& \geq-\alpha f\left(x_{*}\right)+d_{1}^{\prime} m^{2} / f(m) \\
& \geq-c_{1}^{\prime} f\left(x_{*}\right) m^{2} / f(m)+d_{1}^{\prime} m^{2} / f(m) \\
& =\left(d_{1}^{\prime}-c_{1}^{\prime} f\left(x_{*}\right)\right) m^{2} / f(m) .
\end{aligned}
$$

The lemma follows with $d_{2}=d_{1}^{\prime}-c_{1}^{\prime} f\left(x_{*}\right)$.

Before continuing, given $\alpha$ and $\varphi \in K_{\alpha}$ define

$$
\begin{aligned}
& z_{2}=\inf \left\{t>z_{1}+1 \mid x(t)=0\right\}, \quad \text { and } \\
& \delta_{1}=\inf \left\{t>0 \mid x\left(z_{2}+t\right)=x_{*}\right\} .
\end{aligned}
$$

Note that, for $\alpha$ sufficiently large, $x\left(z_{1}+2\right)>x_{*}$, so $z_{2}<\infty$ and $\delta_{1}<1$. In the final lemma of this section we obtain an estimate on $x\left(z_{2}+2\right)$ which will allow us to prove existence of periodic solutions satisfying parts (i) and (iii) of the theorem.

LEMMA 2.6. If $\alpha$ is sufficiently large, $\varphi \in K_{\alpha}$, then there exists a positive constant $d_{3}$ (independent of $\alpha$ and $\varphi \in K_{\alpha}$ ) such that

$$
x\left(z_{2}+2\right) \geq d_{3} m^{2} / f(m) .
$$

ProOF. We first write

$$
\begin{aligned}
x\left(z_{2}+2\right)= & x\left(z_{2}+1\right)-\alpha \int_{0}^{\delta_{1}} f\left(x\left(z_{2}+s\right)\right) d s \\
& -\alpha \int_{\delta_{1}}^{1} f\left(x\left(z_{2}+s\right)\right) d s .
\end{aligned}
$$

Since $z_{1}+1<z_{2}$ and $x(t)$ is increasing on $\left[z_{1}+1, z_{2}+1\right]$, we have

$$
x\left(z_{2}+1\right) \geq x\left(z_{1}+2\right) \geq d_{2} m^{2} / f(m)
$$

( $d_{2}$ as in Lemma 2.5).

Next, note that

$$
-\alpha \int_{0}^{\delta_{1}} f\left(x\left(z_{2}+s\right)\right) d s \geq-\alpha f\left(x_{*}\right) \delta_{1} .
$$

Since $x(t)$ is decreasing on $\left[z_{1}, z_{1}+1\right]$ and $f$ is nondecreasing on $(-\infty, 0]$, it follows that $x(t)$ is concave up on $\left[z_{1}+1, z_{1}+2\right]$. This implies that

$$
x^{\prime}\left(z_{2}\right) \delta_{1} \leq x_{*}, \quad \text { or } \quad \delta_{1} \leq x_{*} / x^{\prime}\left(z_{2}\right) \text {. }
$$

Again using the concavity of $x(t)$ on $\left[z_{1}+1, z_{1}+2\right]$, we observe that

$$
\begin{aligned}
x^{\prime}\left(z_{2}\right) & \geq \frac{\left|x\left(z_{1}+1\right)\right|}{z_{2}-\left(z_{1}+1\right)} \geq\left|x\left(z_{1}+1\right)\right| \\
& \geq c_{2} \int_{0}^{\infty} f(\xi) d \xi / f(m)
\end{aligned}
$$


( $c_{2}$ as in Lemma 2.3). Combining these estimates we conclude that $\delta_{1} \leq c_{3} f(m)$, where $c_{3}=x_{*} /\left(c_{2} \int_{0}^{\infty} f(\xi) d \xi\right)$. Thus,

$$
-\alpha f\left(x_{*}\right) \delta_{1} \geq-c_{3} f\left(x_{*}\right) \alpha f(m) \geq-c_{4} m^{2},
$$

where $c_{4}=c_{3} c_{1} f\left(x_{*}\right)\left(c_{1}\right.$ as in Lemma 2.2).

Next, consider

$$
-\alpha \int_{\delta_{1}}^{1} f\left(x\left(z_{2}+s\right)\right) d s .
$$

It follows, since $x(t)$ is concave up on $\left[z_{2}, z_{1}+2\right]$ and increasing on $\left[z_{2}, z_{2}+1\right]$, that

$$
x\left(z_{2}+s\right) \geq \frac{x\left(z_{2}+1\right)-x_{*}}{1-\delta_{1}}\left(s-\delta_{1}\right)+x_{*}, \quad \delta_{1} \leq s \leq 1 .
$$

$f$ is nonincreasing on $\left[x_{*}, \infty\right)$, so if we substitute

$$
u=\frac{x\left(z_{2}+1\right)-x_{*}}{1-\delta_{1}}\left(s-\delta_{1}\right)+x_{*}
$$

we obtain

$$
\begin{aligned}
-\alpha \int_{\delta_{1}}^{1} f\left(x\left(z_{2}+s\right)\right) d s & \geq \frac{-\alpha\left(1-\delta_{1}\right)}{x\left(z_{2}+1\right)-x_{*}} \int_{x_{*}}^{x\left(z_{2}+1\right)} f(u) d u \\
& \geq \frac{-\alpha\left(1-\delta_{1}\right)}{x\left(z_{2}+1\right)-x_{*}} \int_{x_{*}}^{\infty} f(u) d u
\end{aligned}
$$

Using the fact that $\delta_{1}$ is bounded away from 1 and $x\left(z_{2}+1\right)$ is bounded away from $x_{*}$ for large $\alpha$, it is easy to see that there exists a positive constant $c_{5}$ (independent of $\alpha$ and $\varphi \in K_{\alpha}$ ) such that

$$
\begin{aligned}
-\alpha \int_{\delta_{1}}^{1} f\left(x\left(z_{2}+s\right)\right) d s & \geq-c_{5} \alpha / x\left(z_{2}+1\right) \\
& \geq\left(-c_{5} / d_{2}\right) \alpha f(m) / m^{2} \\
& \geq-c_{5} c_{1} / d_{2} .
\end{aligned}
$$

(Here we have used (2.8) and Lemma 2.2 in the last two steps.)

Lemma 2.6 now follows using (2.7) together with the estimates (2.8) and (2.11).

We can now prove the existence part of our theorem.

Proof of existence of periodic solutions satisfying properties (i) and (iii) of the theorem. For $\alpha$ sufficiently large, $\varphi \in K_{\alpha}$, we have, for $k^{\prime}$ as chosen after Lemma 2.1 ,

$$
x\left(z_{2}+2\right) \geq d_{3} m^{2} / f(m) \geq d_{3} / c_{1} \alpha \geq k^{\prime} \log \alpha .
$$

(Here we have used Lemmas 2.6 and 2.2.)

Define

$$
\tau=\tau(\varphi, \alpha)=\inf \left\{t \geq z_{2}+2 \mid x(t)=k^{\prime} \log \alpha\right\} .
$$

It is easy to see that $\tau<\infty$. Next define a map $F_{\alpha}: K_{\alpha} \rightarrow C[0,1]$, by

$$
\left(F_{\alpha} \varphi\right)(t)=x(\tau-1-t ; \varphi, \alpha) \text {. }
$$

The definition of $\tau$ and the properties of $f$ imply that $F_{\alpha}\left(K_{\alpha}\right) \subseteq K_{\alpha}$. Moreover, standard arguments (see, for example, [9]) imply that $F_{\alpha}$ is continuous, and Ascoli's 
theorem implies that closure $\left(F_{\alpha}\left(K_{\alpha}\right)\right)$ is compact. Therefore, the Schauder fixed point theorem implies that $F_{\alpha}$ has a fixed point $\varphi_{\alpha}$. It follows that

$$
x_{\alpha}(t)=x\left(t+z_{2} ; \varphi_{\alpha}, \alpha\right),
$$

extended periodically to all of $\mathbf{R}$, is a nonconstant periodic solution of (2.1). It is trivial to see that $x_{\alpha}(t)$ satisfies properties (i) and (iii) of the theorem.

3. The estimate on the period. In this sections we will use the same notation as in $\S 2$. The proof of the estimate given in part (ii) of the theorem follows the outline of the proof of Theorem 2.1 in [4]. We refer the reader to that proof for additional details.

Proof of asymptotic estimate given in part (ii) of the theorem. Let $x_{\alpha}(t)$ denote the periodic solution obtained in $\S 2$. Recall that

$$
x_{\alpha}(t)=x\left(t+z_{2} ; \varphi_{\alpha}, \alpha\right), \quad \text { where } \varphi_{\alpha} \in K_{\alpha} .
$$

Therefore, all of the estimates in $\S 2$ hold for $x_{\alpha}(t)$ modulo a time translation. We will need a crude estimate on $x_{\alpha}(3)$ :

$$
\begin{aligned}
x_{\alpha}(3) & \geq x_{\alpha}(2)-\alpha f\left(x_{\alpha}(2)\right) \\
& \geq d_{3} m^{2} / f(m)-\alpha f\left(d_{3} m^{2} / f(m)\right) \\
& \geq d_{3} \frac{m^{2}}{f(m)}-\alpha a_{2} d_{3}^{r} \frac{m^{2 r}}{(f(m))^{r}} e^{-\lambda d_{3} m^{2} / f(m)} .
\end{aligned}
$$

(Here we have used Lemma 2.6 and the properties of $f$.) Using Lemma 2.2 and the properties of $f$ it is easy to see that there exists a positive constant $d_{4}$ (independent of $\alpha$ ) such that

$$
x_{\alpha}(3) \geq d_{4} m^{2} / f(m) \text {. }
$$

Define $t_{0}=\inf \left\{t>2 \mid x_{\alpha}(t)=m\right\} . x_{\alpha}(t)$ is decreasing on $\left[2, t_{0}\right]$, so

$$
x_{\alpha}(t) \leq x_{\alpha}(t-1), \quad 3 \leq t \leq t_{0} .
$$

Since $f$ is nonincreasing on $\left[x_{*}, \infty\right)$, we obtain

$$
-x_{\alpha}^{\prime}(t)=\alpha f\left(x_{\alpha}(t-1)\right) \leq \alpha f\left(x_{\alpha}(t)\right), \quad 3 \leq t \leq t_{0} .
$$

It follows that

$$
\begin{gathered}
-x_{\alpha}^{\prime}(t) \leq a_{2} \alpha\left(x_{\alpha}(t)\right)^{r} \exp \left(-\lambda x_{\alpha}(t)\right), \quad \text { or } \\
-x_{\alpha}^{\prime}(t)\left(x_{\alpha}(t)\right)^{-r} \exp \left(\lambda x_{\alpha}(t)\right) \leq a_{2} \alpha, \quad 3 \leq t \leq t_{0} .
\end{gathered}
$$

Integrating both sides of (3.2) from 3 to $t_{0}$ and substituting $x=x_{\alpha}(t)$ one obtains

$$
\int_{m}^{x_{\alpha}(3)} x^{-r} e^{\lambda x} d x \leq a_{2} \alpha\left(t_{0}-3\right)
$$

Integration by parts gives

$$
\begin{array}{rl}
\int_{m}^{x_{\alpha}(3)} x^{-r} e^{\lambda x} & d x \\
= & \frac{1}{\lambda}\left(x_{\alpha}(3)\right)^{-r} \exp \left(\lambda x_{\alpha}(3)\right)-\frac{1}{\lambda} m^{-r} e^{\lambda m}+\frac{r}{\lambda} \int_{m}^{x_{\alpha}(3)} x^{-r-1} e^{\lambda x} d x .
\end{array}
$$


Using (3.1) it is easy to see that there is a positive constant $d_{5}$ (independent of $\alpha$ ) such that

$$
\begin{aligned}
\int_{m}^{x_{\alpha}(3)} x^{-r} e^{\lambda x} d x & \geq d_{5}\left(\frac{f(m)}{m^{2}}\right)^{r} \exp \left(d_{4} \lambda m^{2} / f(m)\right) \\
& \geq \exp \left(d_{6} m^{2} / f(m)\right) \\
& \geq \exp \left(\left(d_{6} / c_{1}\right) \alpha\right)
\end{aligned}
$$

where $d_{6}<d_{4} \lambda$ and $c_{1}$ is as in Lemma 2.2 .

Combining these estimates we obtain

$$
a_{2} \alpha\left(t_{0}-3\right) \geq \exp \left(\frac{d_{6}}{c_{1}} \alpha\right), \quad \text { or } t_{0} \geq\left(a_{2} \alpha\right)^{-1} \exp \left(\frac{d_{6}}{c_{1}} \alpha\right)+3
$$

Recalling that $q_{\alpha}^{\prime}>q_{\alpha}+1>t_{0}+2$, the desired estimate follows easily.

REMARK 3.1. To find an upper bound on the minimal period of $x_{\alpha}(t)$ we would have to be given more information about $f(x)$ as $x \rightarrow-\infty$.

ACKNOWLEDGement. The author would like to thank Professor Roger Nussbaum for many helpful conversations concerning this paper and in related areas.

\section{REFERENCES}

1. S. A. Chapin, Periodic solutions of some nonlinear differential-delay equations, Ph.D. Dissertation, Rutgers Univ., New Brunswick, N.J., 1983.

2. __ Asymptotic analysis of differential-delay equations and nonuniqueness of periodic solutions, Math. Methods Appl. Sci. 7 (1985), 223-237.

3. _ Periodic solutions of differential-delay equations with more than one delay, Rocky Mountain J. Math. 17 (1987), 555-572.

4. S. A. Chapin and R. D. Nussbaum, Asymptotic estimates for the periods of periodic solutions of a differential-delay equation, Michigan Math. J. 31 (1984), 215-229.

5. W. S. C. Gurney, S. P. Blythe, and R. M. Nisbet, Nicholson's blowflies revisited, Nature 287 (1980), 17-21.

6. M. C. Mackey and L. Glass, Oscillation and chaos in physiological control systems, Science 197 (1977), 287-289.

7. R. D. Nussbaum, Asymptotic analysis of functional differential equations and solutions of long period, Arch. Rational Mech. Anal. 81 (1983), 373-397.

8. __ A global bifurcation theorem with application to functional differential equations, J. Funct. Anal. 19 (1975), 319-338.

9. __ Periodic solutions of nonlinear autonomous functional differential equations, Lecture Notes in Math., vol. 730, Springer-Verlag, New York, 1979, pp. 283-325.

10. _ The range of periods of periodic solutions of $x^{\prime}(t)=-\alpha f(x(t-1))$, J. Math. Anal. Appl. 58 (1977), 280-292.

11. Uniqueness and nonuniqueness for periodic solutions of $x^{\prime}(t)=-g(x(t-1))$, J. Differential Equations 34 (1979), 25-54.

12. H. Peters, Comportment chaotique d'une équation differentielle retardée, C. R. Acad. Sci. Paris Ser. A 290 (1980), 1119-1122.

13. D. Saupe, Global bifurcation of periodic solutions of some autonomous differential delay equations Forschungschwerpunkt Dynamische Systeme, Report No. 71, Univ. Bremen, July 1982.

14. H.-O. Walther, Homoclinic solutions and chaos in $\dot{x}(t)=f(x(t-1))$, Nonlinear Anal. Theory Methods Appl. 5 (1981), 775-788.

15. 79 (1981), 127-140. 